\title{
Prevalencia de síntomas de trastorno por estrés post- traumático en población adulta a dos y diez meses del inicio de la cuarentena por COVID-19
}

\section{Prevalence of symptoms of post-traumatic stress disorder in adult population, two and ten months after the outbreak of COVID-19}

\author{
Carlos Alberto Serrano-Juárez ${ }^{1,2}$, Dalia Sofía Vargas-Cabriada', Miguel Ángel de León-Miranda y \\ Diana Lorena García-Reyes ${ }^{1}$ \\ ${ }^{1}$ Iskalti Atención y Educación Psicológica SC, CDMX, México \\ ${ }^{2}$ Facultad de estudios superiores Iztacala, UNAM, México
}

\section{Resumen}

En 2020, se ha advertido que la prevalencia de síntomas de trastorno por estrés post-traumático (TEPT) podría ser alta en personas que han padecido COVID-19. El objetivo de este trabajo fue explorar la prevalencia de síntomas de TEPT en población adulta tras haber iniciado el confinamiento. Participaron dos grupos; uno de 441 (82 \% mujeres; edad: $M=36.89 ; D T=11.93$ ) y otro de 182 adultos (79 \% mujeres; edad: $M=48.1 ; \quad D T=$ $0.8)$; ambos contestaron un cuestionario en línea elaborado por los autores para valorar la prevalencia de síntomas de TEPT a 2 y 10 meses del inicio del confinamiento. Se realizó un análisis descriptivo y paramétrico para la muestra de 2 meses y para la de 10 meses se realizaron ANCOVAs y un análisis de regresión lineal múltiple. Los resultados no mostraron diferencias a los 2 y 10 meses; sin embargo, revelaron una mayor prevalencia de síntomas de TEPT en personas con antecedentes de COVID-19 la cual además aumenta en adultos menores de 40 años. La participación de los profesionales de la salud mental cobrará mayor importancia en los próximos meses y años para la prevención y tratamiento de este trastorno en población que ha padecido COVID-19.

Palabras clave: Trastorno por estrés post.traumático, COVID-19, ansiedad, pandemia, estrés

\begin{abstract}
In 2020, it was suggested that the prevalence of symptoms of post-traumatic stress disorder (PTSD) could be greater in individuals who have acquired COVID-19. The study aim was to investigate the prevalence of PTSD symptoms in the adult population following the beginning of the COVID- 19 outbreak. Two groups participated; one comprising 441 adults (82\% female; age: $M=36.89 ; S D=11.93)$ and another comprising 182 adults (79\% female; age: $M=48.1$; $\mathrm{SD}=0.8)$. Both groups completed an online questionnaire prepared by the authors to assess the prevalence of PTSD symptoms at 2 and 10 months following the start of the outbreak. A descriptive and parametric analysis was performed for the 2- month sample and ANCOVA and multiple linear regres- sion analysis were performed for the 10-month sample. No differences were found at 2 and 10 months; however, the results showed that there was a higher prevalence of PTSD symptoms in people with a history of COVID-19 and in adults younger than 40 years. The participation of mental health professionals will become of increasing relevance in the future to prevent and treat PTSD in the population with COVID-19.
\end{abstract}

Keywords: Post-traumatic stress disorder, COVID-19, Anxiety, Outbreak, Stress

Cómo citar: Serrano-Juárez, C. A., Vargas-Cabriada, D. S., de León-Miranda, M. A., \& García-Reyes, D. L. (2021). Prevalencia de síntomas de trastorno por estrés post-traumático en población adulta a dos y diez meses del inicio de la cuarentena por COVID-19. Escritos de Psicología - Psychological Writings, 14(2), 63-72. https://doi.org/10.24310/ espsiescpsi.v14i2.12923

Correspondencia: Dr. Carlos Alberto Serrano Juárez, Iskalti Atención y Educación Psicológica SC, Clavería 81-A, Colonia Clavería, CP 02080, Azcapotzalco, CDMX, México. E-mail: carlos@iskalti.com, serranojcarlosa@comunidad.unam.mx. 


\section{Introducción}

El 31 de diciembre del 2019 la Organización Mundial de la Salud (OMS) fue notificada de 44 casos de neumonía vírica en Wuhan, China. Para el 7 de enero del 2020 las autoridades chinas declararon que estos casos fueron originados por un nuevo coronavirus conocido como SARS-CoV-2 lo que provocó que el 11 de marzo del 2020 el COVID-19 fuera declarado como pandemia (Cruz-Pacheco et al., 2020; Organización Mundial de la Salud, 2019).

El primer caso detectado en México fue el 27 de febrero del 2020 y dio inicio a la Fase 1 de pandemia por el COVID-19, en donde los casos confirmados eran importados del extranjero sin casos de contagio local. El 18 de marzo se habían confirmado 118 casos y la Secretaria de Salud informó sobre la primera muerte por COVID-19. El 24 de marzo se entró a la Fase 2 ya que se registraron los primeros contagios locales y como consecuencia se decretó la suspensión de ciertas actividades económicas, recomendando el permanecer en casa a la población en general (Suárez et al.,2020).

El 26 de marzo se tomaron medidas extremas suspendiendo toda clase de actividad no esencial para el gobierno federal, entre ellas las actividades académicas presenciales. Para el 21 de abril se entró en Fase 3 confirmando brotes activos y propagación con más de mil casos, cerrando todas aquellas actividades no esenciales del sector privado, público y social (Suárez et al., 2020).

Estas medidas fueron adoptadas debido a la situación mundial por la que se atravesaba como consecuencia del COVID-19, posteriormente se encontraría que los aspectos más afectados por estas medidas han sido el bienestar físico y psicológico, debido a que muchos de los hábitos o rutinas previas se han tenido que modificar, provocando que otros nuevos emerjan (Lasa et al.,2020).

Los efectos psicológicos que trajo el confinamiento por la pandemia han impactado de manera diferente a la población; sin embargo, varios sectores se han visto en riesgo de sufrir niveles altos de angustia (Emery et al., 2021) y un elevado estrés psicosocial (Sandín et al., 2020).

La situación que se vive por el COVID-19 está produciendo un entorno de mucho estrés para los adultos en diversas formas; por un lado se tiene la preocupación por la salud económica y física de su propia familia y por otro está el aislamiento social. Esta situación se ha visto más agravada para aquellos de bajos ingresos y con antecedentes de problemas de salud mental (Fontanesi et al., 2020).

Teniendo en cuenta que la situación involucraba nuevos retos para los adultos que son progenitores de familia, las complicaciones comenzaron a surgir debido a que tenían que asumir nuevas responsabilidades en cuanto a la educación y en tener que dejar de ejercer el rol de supervisor para pasar a ser quienes la brindan (Soto et al., 2020). Asimismo, el uso de las nuevas tecnologías para asistir a las actividades laborales, la interacción y el desarrollo de habilidades socioemocionales son otros retos que se les han presentado a los adultos (Soto et al., 2020).

De acuerdo con los resultados de Spinelli y Pellino (2020), el confinamiento derivado de la pandemia ha sido una situación muy estresante para todos los adultos que son progenitores de familia, sobre todo para aquellos que tienen que regularlo con su vida personal, trabajo en casa y la educación de sus hijos sin ayuda externa alguna, produciendo efectos negativos en la capacidad de ser cuidadores comprensivos y afectando el bienestar de los niños (Spinelli y Pellino, 2020). Estos autores identificaron que la poca organización dentro de los hogares conduce a niveles de estrés más altos en la crianza durante el confinamiento lo que ha ocasiona una menor regulación emocional en los niños y una menor participación de los progenitores en las actividades de sus hijos.

Además de todos los desencadenantes sociales, académicos y laborales que ha conllevado el confinamiento como factores de estrés, con el avance de la pandemia hay también nuevos casos y un aumento en los contagios, lo cual también podría aumentar los niveles ansiedad, estrés e incluso la prevalencia del trastorno por estrés post-traumático (TEPT).

EI TEPT es un trastorno mental que se manifiesta posterior a la exposición de situaciones que han sido excepcionalmente amenazantes o terroríficos (Bisson et al., 2015; Shalev et al., 2017); estas pueden ser un solo evento o bien perdurar durante cierto tiempo. Este trastorno puede conllevar otros síntomas psiquiátricos y alteraciones sistémicas, como cardiovasculares, musculoesqueléticas, gastrointestinales y/o inmunológicas. Los síntomas incluyen recuerdos intrusivos y persistentes del evento, evitación de estímulos relacionados con el trauma, alteraciones negativas en la cognición (como fallas de atención y memoria), del estado de ánimo y signos fisiológicos como hiperventilación (Bisson et al., 2015).

Las enfermedades causadas por los virus SARS-CoV y MERS-CoV causan infecciones del tracto respiratorio inferior, neumonía grave, en ocasiones lesión pulmonar aguda o síndrome de dificultad respiratoria aguda mortal, daño a otros órganos o tejidos como el tracto gastrointestinal, el bazo, los ganglios linfáticos, el cerebro, los músculos esqueléticos, la tiroides y el corazón (Abdelrahman et al., 2020; Yang et al., 2020), y requieren de la implementación de procedimientos médicos invasivos que 
suelen ser percibidos como traumáticos. Estos síntomas e intervenciones ocasionan una alta incidencia de trastornos psiquiátricos (Kaseda y Levine, 2020) ya que los pacientes lo refieren como una experiencia traumática que aumenta los síntomas y prevalencia de TEPT (Kaseda y Levine, 2020) secundario a estas enfermedades respiratorias.

Por lo tanto, la revisión efectuada sobre los efectos psicológicos secundarios a la pandemia ha identificado que el confinamiento y el COVID-19 podrían traer diferentes efectos adversos psicológicos y psiquiátricos, específicamente, la prevalencia de TEPT. Por lo que el objetivo de este trabajo fue explorar la probable existencia de síntomas de TEPT asociado al COVID-19 en adultos a 2 y 10 meses después de haber iniciado la cuarentena. La hipótesis planteada es que se esperaría un aumento de síntomas de TEPT en adultos que han padecido COVID-19 a 2 y 10 meses de haber iniciado la cuarentena.

\section{Participantes}

\section{Método}

En este estudio participaron 623 adultos mexicanos en total (508 mujeres; 115 hombres) de toda la república mexicana. La muestra de 2 meses después de haber iniciado la cuarentena estuvo conformada por 423 adultos (350 mujeres; 73 hombres) y una media de edad en años de 36.89 (DT=11.93). La muestra de 10 meses estuvo compuesta por 182 adultos (145 mujeres; 37 hombres) y una media de edad en años de 48.1 ( $D T=0.8)$.

\section{Instrumentos}

Inventario de síntomas de TEPT por COVID-19 (I-TEPT-COVID-19). Es un instrumento (Apéndice) elaborado por los autores. Los ítems fueron creados teniendo en cuenta la cuarentena y la enfermedad por COVID-19 y se califican mediante una escala de 5 (Siempre) a 1 (Nunca), dependiendo de la frecuencia con la que han padecido el síntoma. Para la puntuación total de este instrumento se suman las puntuaciones y se obtienen puntuaciones mínimas de 21 y máximas de 105, puntuaciones bajas indican menor prevalencia de síntomas de TEPT.

\section{Procedimiento}

El primer paso de esta investigación fue la elaboración de un instrumento que permitiera identificar la prevalencia de síntomas de TEPT derivados del COVID-19. Se realizaron 32 ítems basados en los criterios del trastorno de estrés post-traumático del Manual de diagnóstico y estadístico de los trastornos mentales [DSM-V, por sus siglas en inglés] (APA, 2014). Los ítems fueron validados a través de un instrumento que indicaba el concepto y criterios del TEPT por 20 expertos en psicología clínica; los jueces debían indicar mediante una escala Likert que iba de "totalmente en desacuerdo" a "totalmente de acuerdo" en que medida los ítems se ajustaban a los criterios de TEPT por COVID-19. Para tomar como válido el ítem debía cumplir con un porcentaje de frecuencia mayor o igual al $60 \%$ para las categorías "de acuerdo" y "totalmente de acuerdo", este procedimiento eliminó un total de 11 ítems. El inventario compuesto por 21 ítems fue cargado a formularios de Google y contestado por una población de 18 años en adelante. El instrumento se respondía de acuerdo a una escala tipo Likert que oscilaba entre 1 (nunca) y 5 (siempre). La escala obtuvo un alto nivel de consistencia interna según lo determinado por un alfa de Cronbach de 0.92 .

Una vez validado y con los datos descriptivos y de confiabilidad del cuestionario, este fue publicado el 1 de mayo de 2020 para recabar datos descriptivos del instrumento e identificar la prevalencia de TEPT a dos meses de haber iniciado la cuarentena; mientras que, para los diez meses posteriores, el cuestionario fue lanzado el 1 de febrero de 2021 con el fin de obtener datos para comparar las puntuaciones de ambas muestras. En los dos tiempos en que se recolectaron los datos, los cuestionarios solo se podían contestar una vez que los participantes aceptaban el consentimiento informado que se ajustaba a los aspectos éticos de la Declaración de Helsinki.

\section{Análisis de datos}

Para el análisis de validez por jueces se realizó un análisis de frecuencias y descriptivo. Para determinar la confiabilidad se obtuvo el alfa de Cronbach. Se hizo una correlación de Pearson entre los ítems pares e impares para corroborar la confiabilidad del instrumento. Los análisis mostraron que la relación era lineal con ambas variables normalmente distribuidas, según lo evaluado por la prueba de ShapiroWilk $p>.05$, y no hubo valores atípicos. Hubo una correlación positiva alta y estadísticamente significativa entre los ítems pares e impares $r(420)=.915, p<.001$.

Se hizo una estratificación de la muestra por edades para identificar diferencias en el nivel de síntomas de TEPT; la cual primero se dividió en tres décadas de vida de acuerdo con el Consejo 
Nacional de Población (2000): adultos jóvenes (18-40 años) $n=252$, adultos maduros (41-60 años) $n=155$ y adultos mayores ( $>60$ años) $n=11$. Se realizó un análisis no paramétrico empleando la prueba Kruskal-Wallis debido a la diferencia en el número de participantes de cada grupo. Posteriormente, se realizaron comparaciones por pares utilizando el procedimiento de Dunn con una corrección de Bonferroni para comparaciones múltiples. Puesto que el análisis post hoc reveló diferencias tan solo entre algunos grupos, se estratificó la muestra en dos grupos y para su comparación se utilizó la prueba $t$ de Student para muestras independientes.

Para comparar los resultados entre los 2 y 10 meses después de haber iniciado el confinamiento se realizó un muestreo aleatorizado de la primera población. Con este procedimiento, las variables demográficas (sexo y tiempo del inicio de la cuarentena) se homogeneizaron entre los grupos; sin embargo, el número de contagios y el porcentaje de contacto con alguien contagiado fueron superiores en el grupo de 10 meses, debido al avance de la pandemia. Se realizó un ANCOVA de una vía tomando la edad como covariable para comparar la puntuación en TEPT entre los grupos de 2 y 10 meses.

Debido que se identificó un aumento del número de contagios en el grupo a los 10 meses, se realizó un análisis ANCOVA de una vía, tomando la edad como covaribale, para identificar si existían mayores puntuaciones de síntomas de TEPT en los participantes que ya habían padecido COVID-19 $(0=N o ; 1=S i)$.

Finalmente, se realizó un análisis de regresión lineal múltiple tomando la edad y el COVID-19 como predictores de la sintomatología de TEPT. Todos los análisis de resultados se hicieron utilizando el programa SPSS 25.

\section{Análisis de resultados a 2 meses}

\section{Resultados}

Se recopilaron 423 participantes para la muestra de 2 meses posterior al inicio de la cuarentena, de la cual el 5.2\% habían tenido contacto con alguien con COVID-19 $n=23$, el $99.8 \%$ no había padecido COVID-19 $n=422$ y el $85.3 \%$ inició la cuarentena en marzo, el $9.7 \%$ en abril y el $5 \%$ seguía sin estarlo. El análisis entre el grupo de adultos jóvenes $(n=252)$ y el grupo de adultos maduros y mayores ( $n=166)$ reveló que no hubo valores atípicos en los datos, según lo evaluado mediante la inspección de un diagrama de caja para valores superiores a 1.5 longitudes de caja desde el borde de la caja y a una igualdad de varianzas evaluada con la prueba de Levene $(p>.05)$. Los puntajes de síntomas de TEPT fueron estadísticamente mayores $t(416)=4.345, p<.001$ en el grupo de adultos jóvenes (edad: $M=51.01 ; D T=16.31$ ) que el grupo de adultos maduros y mayores (edad: $M=44.21 ; D T=14.56$ ). Por lo que a continuación se describen los resultados descriptivos para ambas muestras.

El grupo de adultos jóvenes estuvo compuesto por 205 mujeres y 47 hombres, el 5.6\% había tenido contacto con alguien con COVID-19 y se encontró un participante que ya había padecido la enfermedad. El $87.3 \%$ inició la cuarentena en marzo, el $7.9 \%$ en abril y el $4.8 \%$ seguía sin estarlo. Este grupo obtuvo una puntuación media de 51 ( $D T=16.31$ ), una mediana de 51 y un rango de puntuaciones de 22 a 97.

El grupo de adultos maduros y mayores se conformó por 141 mujeres y 25 hombres, ningún participante había padecido COVID-19, pero el 4.8\% había estado en contacto con alguien y el $81.0 \%$ inició la cuarentena en marzo, el $12.7 \%$ en abril y un $5.4 \%$ seguía sin estarlo. Este grupo obtuvo una puntuación media de 44.21 ( $D T=14.56)$, una mediana de 42 y un rango de puntuaciones de 22 a 82 ; los percentiles de cada muestra se pueden ver en la Tabla 1.

Tabla 1

Percentiles por grupo de edades de 18 a 40 años y mayores de 40 años.

\begin{tabular}{ccc}
\hline Grupo & $\begin{array}{c}\text { Adultos jóvenes } \\
\text { 18-40 años }\end{array}$ & $\begin{array}{c}\text { Adultos maduros y mayores } \\
\text { >40 años }\end{array}$ \\
\hline Percentil & Puntuación Directa & Puntuación Directa \\
\hline 90 & 74 & 66 \\
85 & 70 & 62 \\
70 & 60 & 51 \\
50 & 51 & 42 \\
30 & 40 & 34 \\
15 & 33 & 29 \\
10 & 31 & 27 \\
\hline
\end{tabular}


Análisis de resultados a 10 meses

El grupo de 10 meses estuvo compuesto por 174 participantes (141 mujeres; 33 hombres) con una media de edad de $48.03(D T=10.79)$, el 46\% ya había tenido contacto con alguien con COVID-19 ( $n$ $=80)$ y el $17.6 \%$ había padecido COVID-19 $(n=31)$. El 79.9\% inició la cuarentena en marzo, el 9.8\% en abril y el $10.3 \%$ seguía sin estarlo.

El grupo de 2 meses se compuso por 174 participantes (142 mujeres; 32 hombres) con una media de edad de $38.36(D T=12.18)$, el 3.4\% ya había tenido contacto con alguien con COVID-19 $(n=6)$ y el $0.6 \%$ había padecido COVID-19 $(n=1)$. El 86.2\% inició la cuarentena en marzo, el 10.3\% en abril y el $3.4 \%$ seguía sin estarlo (ver Tabla 2 ).

Tabla 2

Resultados descriptivos de los participantes para el análisis entre la muestra de 2 y 10 meses.

\begin{tabular}{|c|c|c|c|c|c|c|c|}
\hline \multicolumn{2}{|c|}{ Grupo } & \multicolumn{3}{|c|}{2 meses } & \multicolumn{3}{|c|}{10 meses } \\
\hline \multicolumn{2}{|c|}{ Variables } & $\mathrm{N}$ & $\begin{array}{c}\text { Edad } \\
\text { Media (DT) }\end{array}$ & Porcentaje & $\mathrm{N}$ & $\begin{array}{c}\text { Edad } \\
\text { Media (DT) }\end{array}$ & Porcentaje \\
\hline \multirow{2}{*}{ Sexo } & Femenino & 142 & $38.23(12.13)$ & 81.6 & 141 & $48.33(10.68)$ & 81 \\
\hline & Masculino & 32 & $38.93(12.59)$ & 18.4 & 33 & $46.75(11.31)$ & 19 \\
\hline \multirow{2}{*}{ Contacto } & $\mathrm{Si}$ & 6 & $32.33(7.91)$ & 3.4 & 80 & $44.73(10.64)$ & 46 \\
\hline & No & 168 & $38.58(12.27)$ & 96.6 & 94 & $50.84(10.15)$ & 54 \\
\hline \multirow{2}{*}{ Covid } & $\mathrm{Si}$ & 1 & 21 & 0.6 & 31 & $44.54(7.09)$ & 17.8 \\
\hline & No & 173 & $38.46(12.15)$ & 99.4 & 143 & $48.79(11.31)$ & 82.2 \\
\hline
\end{tabular}

Nota: $N=$ Número de participantes; $D T$ = Desviación estándar

Los resultados del ANCOVA no revelaron diferencias significativas $F(1,345)=0.447, p=.51 ; n^{2}$ $=.001$ en el nivel de síntomas de TEPT entre los participantes de 2 meses $(M=47.13 ; D T=1.19)$ y 10 meses $(M=45.96 ; D T=1.19)$ después de que inició la cuarentena; ver Tabla 3.

Tabla 3

Variabilidad y medias no ajustadas y ajustadas de los niveles de síntomas de TEPT a 2 y 10 meses; con la edad como covariable.

\begin{tabular}{|c|c|c|c|c|c|c|c|}
\hline & \multirow[b]{2}{*}{$\mathrm{N}$} & \multicolumn{2}{|c|}{ No ajustada } & \multicolumn{2}{|c|}{ Ajustada } & \multirow[b]{2}{*}{$p$} & \multirow{2}{*}{$\eta^{2}$} \\
\hline & & $M$ & DT & M & DT & & \\
\hline 2 meses & 174 & 48.48 & 16.04 & 47.13 & 1.19 & \multirow{2}{*}{.51} & \multirow{2}{*}{.001} \\
\hline 10 meses & 174 & 44.62 & 14.64 & 45.97 & 1.19 & & \\
\hline
\end{tabular}

Nota: $N=$ Número de participantes; $M=$ Media; $D T=$ Desviación estándar

El ANCOVA de una vía para identificar diferencias entre los participantes que ya habían padecido COVID-19 y los que no, considerando la edad como covariable, reveló diferencias significativas $F$ $(1,171)=19.46, p<.001 ; n^{2}=.102$ entre grupos. El análisis post hoc con un ajuste de Bonferroni, mostró que el grupo con antecedentes de COVID-19 ( $M=54.54 ; D T=2.48)$ presentó mayores síntomas de TEPT en comparación con los que no habían tenido la enfermedad $(M=42.46 ; D T=$ 1.45), con una diferencia media de 12.08 (IC del 95\%, 6.67 a 17.48, $p<.001$ ), ver Tabla 4.

Tabla 4

Variabilidad y medias no ajustadas y ajustadas de los niveles de síntomas de TEPT de participantes con y sin COVID-19; con la edad como covariable.

\begin{tabular}{|c|c|c|c|c|c|c|c|}
\hline & \multirow[b]{2}{*}{$\mathrm{N}$} & \multicolumn{2}{|c|}{ No ajustada } & \multicolumn{2}{|c|}{ Ajustada } & \multirow{2}{*}{$p$} & \multirow{2}{*}{$\eta^{2}$} \\
\hline & & $M$ & DT & $M$ & DT & & \\
\hline Control & 143 & 42.31 & 13.01 & 42.47 & 1.16 & \multirow{2}{*}{$<.001$} & \multirow{2}{*}{.11} \\
\hline COVID-19 & 31 & 55.29 & 17.04 & 54.55 & 2.48 & & \\
\hline
\end{tabular}

Nota: $N=$ Número de participantes, $M=$ Media, $D T=$ Desviación estándar

Finalmente, en el análisis de regresión lineal múltiple para predecir la prevalencia de síntomas de TEPT a partir de la edad y el COVID-19, se identificó que hubo linealidad según lo evaluado por gráficos de regresión parcial y un gráfico de residuos estudentizados contra los valores predichos. Se encontró independencia de los residuos, según lo evaluado por una estadística de Durbin-Watson de 1.943. Hubo homocedasticidad, según se evaluó mediante la inspección visual gráfica de residuos estudentizados contra valores predichos no estandarizado. No se identificó evidencia de multicolinealidad, según lo evaluado por valores de tolerancia superiores a 0.1 , no hubo valores de apalancamiento mayores a 0.2 , y valores para la distancia de Cook por encima de 1 . El modelo de regresión múltiple predijo de manera significativa la prevalencia de síntomas de TEPT $F(2,171)=13.91, p<.001$; adj. 
$R^{2}=.13$. Ambas variables se sumaron de forma estadísticamente significativa a la predicción, $p<, 001$. Los coeficientes de regresión y los errores se pueden encontrar en la Tabla 5.

Tabla 5

Resultados de regresión lineal múltiple para la prevalencia de síntomas de TEPT

\begin{tabular}{|c|c|c|c|c|c|c|c|}
\hline \multirow{2}{*}{ TEPT } & \multirow{2}{*}{ B } & \multicolumn{2}{|c|}{$95 \%$ IC para B } & \multirow{2}{*}{ EE B } & \multirow{2}{*}{$B$} & \multirow{2}{*}{$\mathbf{R}^{2}$} & \multirow{2}{*}{$\Delta \mathbf{R}^{2}$} \\
\hline & & LI & LS & & & & \\
\hline Modelo & & & & & & .14 & $.13^{*}$ \\
\hline Constante & $52.733^{* *}$ & 43.09 & 62.37 & 4.85 & & & \\
\hline Edad & $-.214^{*}$ & -.41 & -.22 & .097 & $-.157^{\star}$ & & \\
\hline COVID-19 & $12.076^{* *}$ & 6.673 & 17.48 & 2.738 & $.316^{* *}$ & & \\
\hline
\end{tabular}

Nota: TEPT = trastorno por estrés post-traumático; Modelo = Método "Intro" en SPSS; B = Coeficiente de regresión no estandarizado; IC = Intervalo de confianza; LI = Límite inferior; LS = Límite superior; $E E B=$ Error estándar del coeficiente; $B=$ Coeficiente estandarizado; $R^{2}=$ Coeficiente de determinación; $\Delta \mathbf{R}^{2}=$ ajustado

${ }^{*} \mathrm{p}<.05{ }^{* \star} \mathrm{p}<.001$

\section{Discusión}

El presente estudio tuvo como objetivo describir y explorar la prevalencia de síntomas de TEPT en adultos a los 2 y 10 meses después de iniciar la cuarentena a través de un cuestionario que valoraba los síntomas de este trastorno ante la enfermedad por COVID-19. Nuestros resultados, al igual que en otras investigaciones y con otras poblaciones (Bo et al., 2020; Macías, 2020; Ozamiz-Etxebarria et al., 2020; Xiang y Wu, 2020), revelaron un aumento de la prevalencia de síntomas de TEPT asociado al padecimiento de la enfermedad.

Tras el análisis de resultados, se encontró que a 10 meses del inicio de la cuarentena hubo un incremento en los síntomas de TEPT en participantes que ya habían padecido COVID-19. Oza- mizEtxebarri et al. (2020), encontraron en población española niveles muy bajos de ansiedad, estrés y depresión a inicios de la pandemia; sin embargo, los síntomas aumentaron conforme el confinamiento, lo cual podría deberse al aumento de contagios y por lo tanto la prevalencia de TEPT.

En un primer momento se especulaba que el incremento de estos síntomas se podría deber a factores sociales como el mismo encierro, el desempleo y las dificultades económicas y académicas de los hijos; lo cual se ha comprobado tiene un impacto en el aumento de niveles de estrés al identificar la falta de recursos para poder realizar de manera eficiente las actividades (Macías, 2020). Macías (2020) encontró que a inicios de la pandemia la población mexicana padecía estrés ante la percepción de la falta de recursos para atender pacientes, el colapso que tendría el sistema de salud, el riesgo de contagio y el distanciamiento social. Sin embargo, en nuestros resultados el haber padecido la enfermedad de COVID-19 y la edad de los participantes tiene una relación directa con la prevalencia de los síntomas de TEPT.

La muestra de 2 meses se caracterizó por pocos contagios de COVID-19, mientras que en la de 10 meses el $32 \%$ habían sido contagiados. Sin embargo, no se encontraron diferencias entre los dos grupos, lo cual podría deberse a que en ambas muestras se tuvieron más participantes sin COVID-19, lo cual impacta directamente sobre las medias de la población. Esto podría implicar que los síntomas de TEPT no han aumentado en la población pero sí lo ha hecho sobre aquellos que han padecido la enfermedad. Hay que tener en cuenta que aquellos estudios en los que se han analizado las consecuencias de las pandemias por coronavirus (Carmassi et al., 2020; Chang y Park, 2020; Main et al., 2011; Salehi et al., 2021) han encontrado que las personas que han padecido estas enfermedades, tanto grave como leve, la consideran un evento traumático (Kaseda y Levine, 2020). Esto concuerda con los resultados aquí obtenidos, ya que identifican al COVID-19 como la principal causa del aumento en la prevalencia de síntomas de TEPT con independencia de la edad de la población.

En personas con COVID-19, Xiang et al. (2020) y Bo et al. (2020) ya habían informado sobre la presencia de TEPT, revelando así un problema de salud mental originado por la pandemia. Algunos autores (Alshehri et al., 2020; Chang y Park, 2020; Palomino-Oré y Huarcaya-Victoria, 2020) han identificado una prevalencia de TEPT que va del $20 \%$ al $40 \%$ en pacientes que padecieron COVID-19. Liang et al. (2020) y Kaseda y Levine (2020) sostienen que la incertidumbre del transcurso de la enfermedad, los procedimientos médicos, el miedo a fallecer y estar al borde de la muerte son factores que influyen para que el COVID-19 sea considerada como una enfermedad que provoca TEPT. Restauri y Sheridan (2020) mencionan que los síntomas en estos pacientes se pueden manifestar en forma de revivir los eventos de los procedimientos médicos, en falta de expresividad emocional y en un aumento de la excitación fisiológica que provoca dificultad para dormir, irritación y problemas para concentrarse. 
Si bien esta investigación reveló que la prevalencia de TEPT en pacientes con COVID-19 ocurre con independencia de la edad, también se identificó que la edad predice la severidad de los síntomas. Los resultados obtenidos concuerdan con la investigación de Reynolds et al. (2016), ya que demostraron que adultos maduros y mayores tienen menores síntomas de TEPT en comparación con los adultos jóvenes. Unido a esto, se ha encontrado que la prevalencia de TEPT también es común en personal de salud con una edad menor a 50 años (Marco et al., 2020). Una posible explicación a este hallazgo es que los adultos maduros y mayores tienen una menor exposición a redes sociales y realizan actividades donde se dirige y optimiza el manejo emocional (Reynolds et al., 2016; Sharma et al., 2020).

Unido a la prevalencia de TEPT en pacientes con COVID-19, no se puede dejar de lado que se ha encontrado que el SARS-COV-2 tiene un 33\% a 40\% de desencadenar síntomas neurológicos, como el delírium (Ezpeleta y García Azorín, 2020), el cual se asocia con mayor cantidad de síntomas de TEPT cuando tiene una duración mayor a 40 días. Estas alteraciones neurológicas también tendrían un efecto directo sobre la cognición, ya que se ha encontrado que las personas que padecen signos neurológicos por COVID-19, el 70\% manifiesta alteraciones neuropsicológicas de leves a severas, específicamente, alteraciones atencionales y del funcionamiento ejecutivo (Beaud et al., 2021). A estas alteraciones neurológicas, neuropsiquiátricas y neuropsicológicas hay que agregar las alteraciones inmunosupresoras que se han encontrado en el TEPT lo que lo convierte en un círculo vicioso que afecta la salud mental de estos pacientes (Liang et al., 2020).

A pesar de que este trabajo identifica un aumento en la prevalencia de síntomas de TEPT en pacientes con COVID-19, presenta algunas limitaciones como no haber usado un instrumento estandarizado en identificar TEPT, sin embargo, no se contaba con uno específico ante la pandemia durante el tiempo en el que se realizó la primera evaluación. Ante esto se recurrió a la necesidad de elaborar uno basado en los criterios de estrés postraumático del DSM-V ajustado a la pandemia por COVID-19, el cual contó con validez de expertos, adecuada consistencia interna y confiabilidad de mitades. Hoy en día se cuenta con algunos instrumentos como la escala de estrés percibido (EEP-10) (Campo-Arias et al., 2020) y el Inventario SISCO del Estrés Académico de pandemia (Macías, 2020) que pueden ser usados en muestras actuales. Otra limitación a señalar es que no se pudo controlar variables que podrían asociarse a la prevalencia de los síntomas, como las sociodemográficas ( $p$. ej. empleo y sueldo), la gravedad de la enfermedad, ocupación laboral y profesión ejercida (p. ej. profesionales sanitarios), antecedentes de trastornos neuropsiquiátricos y eventos traumáticos previos al COVID-19, ya que se ha identificado que estos tienen mayores probabilidades de padecer este trastorno mental (Marco et al., 2020; Restauri y Sheridan, 2020). A pesar de estas limitaciones, esta investigación permite comenzar a tomar medidas de prevención y tratamiento en la salud mental para el regreso a la normalidad de estas personas.

A nuestro conocimiento este es el primer estudio realizado en población mexicana que analiza la prevalencia de TEPT a 2 meses y 10 meses de haber iniciado el confinamiento. El principal hallazgo de esta investigación es que no ha habido cambios en la prevalencia de TEPT en la población general; sin embargo, en personas que han padecido COVID-19 la presencia de estos síntomas es mayor y, además, aumenta si la edad es menor a los 40 años. La preocupación que se vive día a día al no poder ver una fecha fija próxima en la que la situación cambie, la exposición a redes sociales de información falsa y el contar con menos estrategias de afrontamiento al estrés podría seguir impactando sobre la prevalencia de estos síntomas teniendo como consecuencias alteraciones en el sueño, alimentación y mala regulación de las emociones. Por lo tanto, aunado a los hallazgos neuropsicológicos y emocionales encontrados en pacientes con COVID-19 es importante tomar en cuenta la prevalencia de TEPT. Esto implica que los profesionales de la salud mental cobran una importancia mayor para el diagnóstico y tratamiento de los efectos emocionales y cognitivos secundarios al COVID-19 ya que el uso de intervenciones cognitivo-conductuales y farmacológica han demostrado ser efectivas para el manejo del TEPT (Qi et al., 2016).

\section{Referencias}

1. Abdelrahman, Z., Li, M., \& Wang, X. (2020). Comparative Review of SARS-CoV-2, SARS-CoV, MERS-CoV, and Influenza A Respiratory Viruses. Frontiers in Immunology, 11, 2309. https://doi. org/10.3389/fimmu.2020.552909

2. Alshehri, F. S., Alatawi, Y., Alghamdi, B. S., Alhifany, A. A., \& Alharbi, A. (2020). Prevalence of post-traumatic stress disorder during the COVID-19 pandemic in Saudi Arabia. Saudi Pharmaceutical Journal, 28(12), 1666-1673. https://doi.org/10.1016/j.jsps.2020.10.013

3. American Psychiatric Association, C. A. (2014). Manual diagnóstico y estadístico de los trastornos mentales: DSM-5. Editorial médica panamericana. 
4. Beaud, V., Crottaz-Herbette, S., Dunet, V., Vaucher, J., Bernard-Valnet, R., Du Pasquier, R., Bart, P.-A., \& Clarke, S. (2021). Pattern of cognitive deficits in severe COVID-19. Journal of Neurology, Neurosurgery \& Psychiatry, 92(5), 567-568. https://doi.org/10.1136/jnnp-2020-325173

5. Bisson, J. I., Cosgrove, S., Lewis, C., \& Robert, N. P. (2015). Post-traumatic stress disorder. BMJ (Clinical Research Ed.), 351, h6161-h6161. PubMed. https://doi.org/10.1136/bmj.h6161

6. Bo, H., Li, W., Yang, Y., Wang, Y., Zhang, Q.-E., Cheung, T., Wu, X.-J., \& Xiang, Y. (2020). Posttraumatic stress symptoms and attitude toward crisis mental health services among clinically stable patients with COVID-19 in China. Psychological Medicine, 1-2. https://doi.org/10.1017/ S0033291720000999

7. Campo-Arias, A., Pedrozo-Cortés, M. J., \& Pedrozo-Pupo, J. C. (2020). Pandemic-Related Perceived Stress Scale of COVID-19: An exploration of online psychometric performance. Revista Colombiana de Psiquiatria (English Ed.), 49(4), 229-230. PubMed. https://doi.org/10.1016/i. rcp.2020.05.005

8. Carmassi, C., Foghi, C., Dell'Oste, V., Cordone, A., Bertelloni, C. A., Bui, E., \& Dell'Osso, L. (2020). PTSD symptoms in healthcare workers facing the three coronavirus outbreaks: What can we expect after the COVID-19 pandemic. Psychiatry Research, 292, 113312. https://doi. org/10.1016/i.psychres.2020.113312

9. Chang, M. C., \& Park, D. (2020). Incidence of Post-Traumatic Stress Disorder after Coronavirus Disease. Healthcare, 8(4), Article 4. https://doi.org/10.3390/healthcare8040373

10. Consejo Nacional de Población. (2000). Índices de desarrollo social en las etapas del curso de vida. México, CONAPO. https://bit.ly/2VYq9Wy

11. Cruz-Pacheco, G., Bustamante-Castañeda, J. F., Caputo, J. G., Jiménez-Corona, M. E., \& Ponce-de-León-Rosales, S. (2020). Dispersion of a new coronavirus SARS-CoV-2 by airlines in 2020: Temporal estimates of the outbreak in Mexico. Revista de investigación clínica, 72(3), 138-143. https://doi.org/10.24875/RIC.20000113

12. Emery, R. L., Johnson, S. T., Simone, M., Loth, K. A., Berge, J. M., \& Neumark-Sztainer, D. (2021). Understanding the impact of the COVID-19 pandemic on stress, mood, and substance use among young adults in the greater Minneapolis-St. Paul area: Findings from project EAT. Social Science \& Medicine, 276, 113826. https://doi.org/10.1016/i.socscimed.2021.113826

13. Ezpeleta, D., \& García Azorín, D. (2020). Manual COVID-19 para el neurólogo general. Madrid: Ediciones SEN, 78.

14. Fontanesi, L., Marchetti, D., Mazza, C., Di Giandomenico, S., Roma, P., \& Verrocchio, M. C. (2020). The effect of the COVID-19 lockdown on parents: A call to adopt urgent measures. Psychological Trauma: Theory, Research, Practice, and Policy, 12(S1), S79-S81. https://doi. org/10.1037/tra0000672

15. Kaseda, E. T., \& Levine, A. J. (2020). Post-traumatic stress disorder: A differential diagnostic consideration for COVID-19 survivors. The Clinical Neuropsychologist, 34(7-8), 1498-1514. https:// doi.org/10.1080/13854046.2020.1811894

16. Lasa, N. B., Benito, J. G., Montesinos, D., Manterola, D., Sánchez, D., García, D., \& Germán, D. (2020). Las consecuencias psicológicas de la COVID-19 y el confinamiento. Servicio Publicaciones de la Universidad del País Vasco. https://n9.cl/zoygd

17. Liang, L., Gao, T., Ren, H., Cao, R., Qin, Z., Hu, Y., Li, C., \& Mei, S. (2020). Post-traumatic stress disorder and psychological distress in Chinese youths following the COVID-19 emergency. Journal of Health Psychology, 25(9), 1164-1175. https://doi.org/10.1177/1359105320937057

18. Liang, X., Zhu, Y., \& Fang, Y. (2020). COVID-19 and post-traumatic stress disorder: A vicious circle involving immunosuppression. CNS Neuroscience \& Therapeutics, 26(8), 876-878. PubMed. https://doi.org/10.1111/cns.13431

19. Macías, A. B. (2020). El estrés de pandemia (COVID 19) en población mexicana. http://www.upd. edu.mx/PDF/Libros/Coronavirus.pdf

20. Main, A., Zhou, Q., Ma, Y., Luecken, L. J., \& Liu, X. (2011). Relations of SARS-related stressors and coping to Chinese college students' psychological adjustment during the 2003 Beijing SARS epidemic. Journal of Counseling Psychology, 58(3), 410-423. https://doi.org/10.1037/a0023632

21. Marco, C. A., Larkin, G. L., Feeser, V. R., Monti, J. E., Vearrier, L., \& for the ACEP Ethics Committee. (2020). Post-traumatic stress and stress disorders during the COVID-19 pandemic: Survey of emergency physicians. Journal of the American College of Emergency Physicians Open, 1(6), 1594-1601. https://doi.org/10.1002/emp2.12305 
22. Organización Mundial de la Salud. (2019). Información básica sobre la COVID-19. https:// www.who.int/es/emergencies/diseases/novel-coronavirus-2019?gclid=CjwKCAjwidOIBhA EiwAHz8xm1JFzw6IVIlgFZ7ynwezUzWv BkPILI1-5VyWU1Vt809Lftdt7Oc4xoCIF8QAvD BwE

23. Ozamiz-Etxebarria, N., Dosil-Santamaria, M., Picaza-Gorrochategui, M., \& Idoiaga-Mondragon, N. (2020). Niveles de estrés, ansiedad y depresión en la primera fase del brote del COVID-19 en una muestra recogida en el norte de España. Cadernos de Saúde Pública, 36, e00054020. https://doi.org/10.1590/0102-311X00054020

24. Palomino-Oré, C., \& Huarcaya-Victoria, J. (2020). Trastornos por estrés debido a la cuarentena durante la pandemia por la COVID-19. Horizonte Médico (Lima), 20. http://dx.doi.org/10.24265/ horizmed.2020.v20n4.10

25. Qi, W., Gevonden, M., \& Shalev, A. (2016). Prevention of Post-Traumatic Stress Disorder After Trauma: Current Evidence and Future Directions. Current Psychiatry Reports, 18(2), 20-20. PubMed. https://doi.org/10.1007/s11920-015-0655-0

26. Restauri, N., \& Sheridan, A. D. (2020). Burnout and Posttraumatic Stress Disorder in the Coronavirus Disease 2019 (COVID-19) Pandemic: Intersection, Impact, and Interventions. Journal of the American College of Radiology : JACR, 17(7), 921-926. PubMed. https://doi.org/10.1016/j. jacr.2020.05.021

27. Reynolds, K., Pietrzak, R. H., Mackenzie, C. S., Chou, K. L., \& Sareen, J. (2016). Post-Traumatic Stress Disorder Across the Adult Lifespan: Findings From a Nationally Representative Survey. The American Journal of Geriatric Psychiatry, 24(1), 81-93. https://doi.org/10.1016/j.jagp.2015.11.001

28. Salehi, M., Amanat, M., Mohammadi, M., Salmanian, M., Rezaei, N., Saghazadeh, A., \& Garakani, A. (2021). The prevalence of post-traumatic stress disorder related symptoms in Coronavirus outbreaks: A systematic-review and meta-analysis. Journal of Affective Disorders, 282, 527-538. PubMed. https://doi.org/10.1016/i.jad.2020.12.188

29. Sandín, B., Valiente, R. M., García-Escalera, J., \& Chorot, P. (2020). Impacto psicológico de la pandemia de COVID-19: Efectos negativos y positivos en población española asociados al periodo de confinamiento nacional. Revista de Psicopatología y Psicología Clínica, 25(1), Article 1. https://doi.org/10.5944/rppc.27569

30. Shalev, A., Liberzon, I., \& Marmar, C. (2017). Post-Traumatic Stress Disorder. New England Journal of Medicine, 376(25), 2459-2469. https://doi.org/10.1056/NEJMra1612499

31. Sharma, V., Reina Ortiz, M., \& Sharma, N. (2020). Risk and Protective Factors for Adolescent and Young Adult Mental Health Within the Context of COVID-19: A Perspective From Nepal. The Journal of Adolescent Health : Official Publication of the Society for Adolescent Medicine, 67(1), 135-137. PubMed. https://doi.org/10.1016/j.jadohealth.2020.04.006

32. Soto, M. A. V., Moreno, W. T.B., \& Rosales, L. Y.A. (2020). La educación fuera de la escuela en época de pandemia por Covid 19. Experiencias de alumnos y padres de familia. Revista electrónica sobre cuerpos académicos y grupos de investigación, 7(14),111-134

33. Spinelli, A., \& Pellino, G. (2020). COVID-19 pandemic: Perspectives on an unfolding crisis. British Journal of Surgery, 107(7), 785-787. https://doi.org/10.1002/bjs.11627

34. Suárez, V., Suarez Quezada, M., Oros Ruiz, S., \& Ronquillo De Jesús, E. (2020). Epidemiology of COVID-19 in Mexico: From the 27th of February to the 30th of April 2020. Revista clínica española, 220(8), 463-471. PubMed. https://doi.org/10.1016/j.rce.2020.05.007

35. Xiang, Y.-T., Yang, Y., Li, W., Zhang, L., Zhang, Q., Cheung, T., \& Ng, C. H. (2020). Timely mental health care for the 2019 novel coronavirus outbreak is urgently needed. The Lancet Psychiatry, 7(3), 228-229. https://doi.org/10.1016/S2215-0366(20)30046-8

36. Yang, Y., Peng, F., Wang, R., Yange, M., Guan, K., Jiang, T., Xu, G., Sun, J., \& Chang, C. (2020). The deadly coronaviruses: The 2003 SARS pandemic and the 2020 novel coronavirus epidemic in China. Journal of Autoimmunity, 109, 102434-102434. PubMed. https://doi.org/10.1016/i. jaut.2020.102434 
Inventario de síntomas de TEPT por COVID-19

\section{Apéndice}

Instrucciones: A continuación encontrarás algunos enunciados que tienen el objetivo de valorar tu estado emocional ante la situación actual, te pedimos marcar con un " $X$ " con qué frecuencia te han ocurrido las siguientes afirmaciones a partir de que inició la cuarentena por COVID-19. Ten en cuenta la siguiente escala:

Nunca $=1$

Poco $=2$

Algunas veces $=3$

Frecuentemente $=4$

Siempre $=5$

\begin{tabular}{|c|c|c|c|c|c|c|}
\hline \multicolumn{7}{|c|}{ Ítem } \\
\hline 1 & Me preocupa en exceso el poder enfermarme & 1 & 2 & 3 & 4 & 5 \\
\hline 2 & Me cuesta trabajo controlar mi respiración & 1 & 2 & 3 & 4 & 5 \\
\hline 3 & Tengo dificultades para controlar mi preocupación & 1 & 2 & 3 & 4 & 5 \\
\hline 4 & Siento que me falta la respiración & 1 & 2 & 3 & 4 & 5 \\
\hline 5 & Tengo inquietud y preocupación de estar encerrado & 1 & 2 & 3 & 4 & 5 \\
\hline 6 & Tengo dificultades para concentrarme en mis actividades & 1 & 2 & 3 & 4 & 5 \\
\hline 7 & Estoy irritable & 1 & 2 & 3 & 4 & 5 \\
\hline 8 & Me enojo fácilmente & 1 & 2 & 3 & 4 & 5 \\
\hline 9 & Me cuesta trabajo poder dormir por las noches & 1 & 2 & 3 & 4 & 5 \\
\hline 10 & Me despierto constantemente por las noches & 1 & 2 & 3 & 4 & 5 \\
\hline 11 & Me siento cansado o cansada por la mañana después de despertar & 1 & 2 & 3 & 4 & 5 \\
\hline 12 & Tengo pesadillas recurrentemente ( 4 a 5 veces a la semana) & 1 & 2 & 3 & 4 & 5 \\
\hline 13 & Mi estado emocional actual ha afectado mi vida diaria & 1 & 2 & 3 & 4 & 5 \\
\hline 14 & Tengo problemas para controlar mi miedo & 1 & 2 & 3 & 4 & 5 \\
\hline 15 & Me siento nervioso aun cuando no ha ocurrido nada & 1 & 2 & 3 & 4 & 5 \\
\hline 16 & Siento un vacío y/o desesperanza & 1 & 2 & 3 & 4 & 5 \\
\hline 17 & Tengo poco interés por hacer mis actividades & 1 & 2 & 3 & 4 & 5 \\
\hline 18 & Me cuesta disfrutar de mis actividades diarias & 1 & 2 & 3 & 4 & 5 \\
\hline 19 & He perdido un peso considerable en las últimas dos semanas & 1 & 2 & 3 & 4 & 5 \\
\hline 20 & Me siento poco útil para hacer mis actividades & 1 & 2 & 3 & 4 & 5 \\
\hline 21 & Tengo pensamientos suicidas & 1 & 2 & 3 & 4 & 5 \\
\hline
\end{tabular}

Notas de autor

Conflicto de intereses. Los autores declaran no tener ningún conflicto de interés.

RECIBIDO: 29 de junio de 2021

MODIFICADO: 15 de septiembre de 2021

ACEPTADO: 16 de septiembre de 2021 